OPEN ACCESS

Edited by: Holmes Finch,

Ball State University, United States

Reviewed by:

Evgueni Borokhovski,

Concordia University, Canada

Paul T. Barrett,

Advanced Projects R\&D Ltd.,

New Zealand

${ }^{*}$ Correspondence:

Frank Zenker

frank.zenker@fil.Iu.se

Specialty section:

This article was submitted to

Quantitative Psychology

and Measurement,

a section of the journal

Frontiers in Psychology

Received: 22 September 2016 Accepted: 04 October 2017 Published: 27 October 2017

Citation:

Witte EH and Zenker F (2017) From

Discovery to Justification: Outline of an Ideal Research Program

in Empirical Psychology.

Front. Psychol. 8:1847.

doi: 10.3389/fpsyg.2017.01847

\section{From Discovery to Justification: Outline of an Ideal Research Program in Empirical Psychology}

\author{
Erich H. Witte ${ }^{1}$ and Frank Zenker ${ }^{2,3,4,5 *}$ \\ ${ }^{1}$ Social and Economic Psychology, University of Hamburg, Hamburg, Germany, ${ }^{2}$ Philosophy and Cognitive Science, Lund \\ University, Lund, Sweden, ${ }^{3}$ Institute of Philosophy, Slovak Academy of Sciences (SAS), Bratislava, Slovakia, ${ }^{4}$ Philosophy, \\ Konstanz University, Konstanz, Germany, ${ }^{5}$ Institute of Logic and Cognition, Sun Yat-sen University, Guangzhou, China
}

The gold standard for an empirical science is the replicability of its research results. But the estimated average replicability rate of key-effects that top-tier psychology journals report falls between 36 and 39\% (objective vs. subjective rate; Open Science Collaboration, 2015). So the standard mode of applying null-hypothesis significance testing (NHST) fails to adequately separate stable from random effects. Therefore, NHST does not fully convince as a statistical inference strategy. We argue that the replicability crisis is "home-made" because more sophisticated strategies can deliver results the successful replication of which is sufficiently probable. Thus, we can overcome the replicability crisis by integrating empirical results into genuine research programs. Instead of continuing to narrowly evaluate only the stability of data against random fluctuations (discovery context), such programs evaluate rival hypotheses against stable data (justification context).

Keywords: confirmation, knowledge accumulation, meta-analysis, psi-hypothesis, replicability crisis, research programs, significance-test, test-power

\section{INTRODUCTION}

Empirical psychology and the social sciences at large remain in crisis today, because (too) many key-results cannot be replicated (Baker, 2015; Open Science Collaboration, 2015; Etz and Vandekerckhove, 2016). Having diagnosed a disciplinary crisis as early as Willy (1889), psychologists did so most recently in a special issue of Perspectives on Psychological Science (Witte, 1996a; Pashler and Wagenmakers, 2012; Spellman, 2012; Sturm and Mülberger, 2012). Particularly the crisis of significance-testing is about as old as the test itself (Witte, 1980; Cowles, 1989; Harlow et al., 1997). Setting the current crisis apart is the insight that null-hypothesis significance testing (NHST) has broadly failed to deliver the stable effects that should characterize empirical knowledge. Many researchers are therefore (rightly) concerned that all published effects are under doubt. The perhaps most pressing question today is how our field might regain trust.

In our view, the ongoing replicability crisis reflects a goal-conflict between publishing statistically significant results as an individual researcher and increasing the trustworthiness of scientific knowledge as a community (Bakker et al., 2012; Nosek et al., 2012; Ioannidis, 2014).

\footnotetext{
${ }^{1}$ In fact, OSC reported an average replicability-rate of some $36 \%$. Worse still, a Bayesian approach finds clear and consistent results in merely $11 \%$ of 72 reanalyzed datasets (Etz and Vandekerckhove, 2016). Though Gilbert et al. (2016, p. 1037-a) submit that "[i]f OSC (Open Science Collaboration) had limited their analyses to endorsed studies, they would have found $59.7 \%$ [ $95 \%$ confidence interval: $47.5,70.9 \%$ ] were replicated successfully," it is clear that even $59.7 \%$ is insufficient to regain trust.
} 
Acknowledging that we can separate the corresponding research activities only analytically, we map both goals onto the terms 'discovery' and 'justification' (aka 'DJ-distinction'). Since the status quo favors "making discoveries," we submit, the balance between these goals must be redressed. As regards variously proposed "minimally invasive" remedies, however, we find that such "soft" measures are insufficient to regain trust. ${ }^{2}$

We rest our case on the observation that psychologists typically deploy statistical inference methods in underpowered studies (Maxwell, 2004). This praxis generates theoretically disconnected "one-off" discoveries whose replication is improbable. But such results should not be trusted, because they are insufficiently stable to justify, or corroborate, a theoretical hypothesis (Rosnow and Rosenthal, 1989). By contrast, corroboration is possible within a research program. To overcome the crisis, therefore, our community should come to coordinate itself on joint long-term research endeavors.

\section{FROM DISCOVERY TO JUSTIFICATION}

\section{Overview}

Constructing a psychological theory begins with discovering non-random relations between antecedent variables and their (causal) consequences, aka stable effects. Relying on a probabilistic version of the lean DJ-distinction, this section contrasts the discovery and the justification context, explains the replicability of empirical results, and shows why underpowered discoveries cannot be trusted. We then define two key concepts: induction quality of data and corroboration quality of hypotheses, and formulate a brief upshot.

\section{Stable Effects}

As late 19th-century psychologists transformed their field into an empirical science, the guiding idea was to base empirical hypotheses on stable non-random effects. Indeed, only stable effects guide researchers toward the explananda. Otherwise, explanation would be pointless-for what should be explained? This pedestrian insight makes the discovery of a stable effect as necessary as the managing of random influences is regularly unavoidable (e.g., in measurement, sampling, or situation-construction). We therefore discover an effect but in a probabilistic sense.

As we thus evaluate our chances of having made a discovery, we must gauge the effect's deviation from random against a statistical significance threshold. Of course, if we cannot discover an effect with certainty, then it follows by parity of reasoning that we cannot falsify it with certainty either. We must therefore ever invest some trust that the effect in fact surpasses potential random influences.

\footnotetext{
${ }^{2}$ As a recent survey indicates, psychologists are open to milder changes (Fuchs et al., 2012). The accepted rules of best research practice, for instance, shall not be turned into binding publication conditions. Moreover, $84 \%$ among respondents find that reviewers should be more tolerant of imperfections in what their peers submit for publication (ibid., p. 640). Additional proposals include intensifying communication, pre-registering hypotheses, and exchanging data- and designcharacteristics (e.g., Nosek and Bar-Anan, 2012; Nosek et al., 2015).
}

Also known as 'stable observations', such effects register as highly probable deviations from a content-free random or nullhypothesis $\left(\mathrm{H}_{0}\right)$. But even a stable effect (in this probabilistic sense) may be subsumed under distinct explanatory hypotheses. We must therefore corroborate such diverging explanations via a theory that predicts the effect's probabilistic signature from initial and boundary conditions.

If we base hypothesis construction and validation on uncertain observation, then hypothesis corroboration likewise entails the probabilistic comparison of a hypotheses pair. Pace efforts by the likes of Rudolf Carnap and Karl Popper, this is an immediate consequence of recognizing that uncontrollable random influences are relevant to theory acceptance. As we now show, it is this interplay between an effect's probabilistic discovery and its probabilistic corroboration that connects the discovery and the justification contexts (aka DJ-distinction).

\section{The Lean DJ-Distinction}

Discovery and justification are fairly self-evident concepts. The dominant mode of deploying statistical inference methods nevertheless reflects them inadequately. Indeed, a review of the textbook literature would show that the NHST approach largely ignores the DJ-distinction. To set this right, we rely on a probabilistic model to make a non-controversial version of the DJ-distinction precise. ${ }^{3}$ Hoyningen-Huene (2006) calls it the 'lean DJ-distinction' to denote "an abstract distinction between the factual [...] and the normative or evaluative [...]" (ibid., p. 128).

In adapting this distinction, 'discovery' refers to research activities in the data space that employ probabilities, while 'justification' denotes activities in the hypotheses space that employ likelihoods. Unlike a measure of the probability, $P$, of data given a hypothesis [where $0 \leq P(\mathrm{D}, \mathrm{H}) \leq 1$ ], likelihoods, $L$, aka 'inverse probabilities', are a sort of probability measure for hypotheses given data [where $0<L(\mathrm{H} \mid \mathrm{D})<\infty$ ]. 'Data space' and 'hypotheses space' are analytical constructs, respectively denoting the collection of stable data and their subsumption under hypotheses.

Of course, research activities alternate between both spaces, witness metaphorical ideas, and such practices as data"torturing" or inventing ad hoc hypotheses, etc. Similar heuristics are fine, but they do not amount to a hypothesis test. After all, discovery context activities focus on phenomena we are yet to discover. So a probabilistic model defines a discovery as a (theory-laden) observation of a non-random effect. This yields as relevant elements the $\mathrm{H}_{0}$-hypothesis and the actual data distribution. If data have a low probability given the $\mathrm{H}_{0}$ (aka the effect's $p$-value; see Fisher, 1956), this is called a 'discovered effect.'

But when we next evaluate the trustworthiness of data, the crucial question is this: if we treat the data distribution that

\footnotetext{
${ }^{3}$ Introduced by Reichenbach (1938), the DJ-distinction has been contended from diverse perspectives, notably since the reception of Kuhn (1970). Extant discussion of the distinction's multiple versions questions the separability of both contexts on temporal, methodological, goal- or question-related criteria. See Schickore and Steinle (2006) and references provided there.
} 
had been obtained in a given test-condition as a theoretical parameter, and moreover hold constant the $p$-value and the number of observations, what is the probability of obtaining the same distribution given the $\mathrm{H}_{0}$ in a subsequent instance of this test-condition? ${ }^{4}$ So to assess the trustworthiness of data is to measure the probability of replicating a non-random effect. This leads us to consider test-power.

\section{Test-Power and Statistical Significance vs. Theoretical Importance}

Cohen (1962) had suggested that empirical studies in the social sciences tend to be underpowered. This entails a low probability of successfully replicating a non-random effect. His guiding assumption was that the true amount of influence from independent onto dependent variables be of medium size $(d=0.50)$.

Provided a typical sample size around $\mathrm{n} 1=\mathrm{n} 2=30$, if we assume $d=0.50$ and a two-sided $\alpha$-error $=0.05$, then average test-power comes to $1-\beta$-error $=0.46$. Some 25 years later, Sedlmeier and Gigerenzer (1989) arrived at a similar value. (The $\alpha$-error denotes the chance of obtaining a false positive test-result, the $\beta$-error the chance of obtaining a false negative result; both errors are normally non-zero, and should be small for data to be trustworthy.) Test-power $=0.46$ implies that samples are typically too small to expect a stable effect. Therefore, empirical results obtained with similar testpower may at best issue invitations to more closely study such "discoveries."

In psychology as elsewhere in the social sciences, however, researchers tend to over-report such underpowered results as hypothesis confirmations. Already some 50 years ago, this praxis was identified as a cause of publication bias (Sterling, 1959). To more fully appreciate why this overstates the capabilities of the method applied, consider that NHST does normally not specify the $\mathrm{H}_{1}$. One thus fails to assign precise semantic content to it. By contrast, the (random) $\mathrm{H}_{0}$ does tend to be well-specified. ${ }^{5}$

If data now display a sufficiently large deviation from random, this is (erroneously) interpreted as a discovery of theoretical importance. But on the reasonable assumption that theoretically important discoveries are stable rather than random, a necessary condition in order to meaningfully speak of a theoretically important discovery is to have

\footnotetext{
${ }^{4}$ The inferential strategy generating this question entails an inductive transition. After all, by formulating a specific non-random hypothesis the probability model, as it were, transforms "observational" data into a quasi-theoretical hypothesis. So we run into the unsolved issue of justifying induction as a valid inference (see below).

${ }^{5}$ This praxis is not restricted to NHST but also implicates proponents of Bayesfactor testing. Verhagen and Wagenmakers (2014, p. 1461), for instance, state that "[t]he problem with this analysis [likelihood-testing] is that the exact alternative effect size $\delta_{a}$ is never known beforehand. In Bayesian statistics, this uncertainty about $\delta$ is addressed by assigning it a prior distribution." Indeed, "[t]he major drawback of this procedure is that it is based on a point estimate, thereby ignoring the precision with which the effect size is estimated" (ibid., p. 1463). Similarly, "[w]e assumed that the alternative was at a single point. This assumption, however, is too restrictive to be practical" (Rouder et al., 2009, p. 229).
}

properly employed a reproducibility measure such as testpower. This in turn presupposes a specified effect size, which the standard mode of deploying NHST, however, cannot offer. So NHST cannot warrant an immediate transition from 'statistically significant effect' to 'theoretically important discovery.'

Many researchers appear to be satisfied knowing that testpower is maximal, but fail to check its exact value. So they do not properly deploy a reproducibility measure. The one "good" reason for this is their failure to specify the $\mathrm{H}_{1}$, because only its specification renders test-power quantifiable. This failure contributes to the confidence loss in our research results. Understandably so, too, for some 50 years after Cohen had hinted at $d=0.46$, test-power typically registers even lower, at $d=0.35$. So most studies are underpowered (Bakker et al., 2012; Baker, 2015).

Against the background of our probabilistic model, we proceed to explain why so many empirical results should not be trusted.

\section{Trustworthy Discoveries}

In his influential textbook Cohen had recommended that " $[w]$ hen the investigator has no other basis for setting the desired power value, the value $[1-\beta$-error $=0.80]$ is used" (Cohen, 1977, p. 56). This set a widely accepted standard. Together with $\alpha=0.05$, it implies a weighing of epistemological values that makes the preliminarily discovery of an effect four times more important than its stable replication. (This alone goes some way toward explaining $1-\beta$-error $=0.35$.) Similarly-powered "discoveries" thus are typically instable.

It follows that few effects which arise in the discovery context are known to deserve a theoretical explanation. Hence, the unsophisticated application of NHST as a discovery method regularly fails to inform the evaluation of hypotheses in the justification context. Since justification contexts activities aim at developing the comparatively best-corroborated hypotheses into theories, it can hardly surprise that psychology offers so few genuine theories (we return to this in Section "Precise Theoretical Constructs?").

To set this right, discovery context activities must establish non-random effects that also feature a high replication probability, i.e., stable effects. Yet, the current researchand publication-praxis does not fully reflect that insight. For instance, though "mere" replications do serve to evaluate whether an effect is stable, until recently one could not publish such work in a top-tier journal (see Holcombe, 2016 on Perspectives on Psychological Science's new replication section).

In summary, we can quantify the replication probability of data only if we specify two point hypotheses $\left(\mathrm{H}_{0}, \mathrm{H}_{1}\right)$. To improve the trustworthiness of empirical knowledge, we must therefore increase the precision of theoretical assumptions (Klein, 2014). For only this yields knowledge of test-power, and only then has the comparative corroboration of the $\mathrm{H}_{1}$ by a data-set $\mathrm{D}$ (as compared to a rival $\mathrm{H}_{0}$ ) not been indirectly deduced from our estimation of $\mathrm{D}$ given the $\mathrm{H}_{0}$.

This puts us in a position to offer two central definitions. 


\section{Induction Quality of Data, Corroboration Quality of Hypotheses}

Our probabilified version of the lean DJ-distinction suggests that two analytically distinct activities govern the research process. Roughly, one first creates an empirical set-up serving as a testcondition to obtain data of sufficient induction quality (discovery context). Next, one tests point-hypotheses against such data (justification context).

In more detail, discovery context activities evaluate data by means of descriptive and inferential statistics given fixed hypotheses. Since this gauges induction quality of data, we perform an evaluation in the data space. (In fact, we proceed hypothetically, effectively assessing if data would be sufficiently trustworthy if they were obtained.) Exactly this is expressed by 'gauging the probability that data are replicable given two point-hypotheses.'

Proceeding to the justification context, we now evaluate pointhypotheses in order to gauge corroboration quality of hypotheses given data. So we perform an evaluation in the hypotheses space. Crucially, only if we in fact obtain data of sufficient induction quality can we properly quantify the inductive support that actual data lend to a hypothesis. So 'gauging corroboration quality' refers to evaluating the degree to which probably replicable data support one hypothesis more than another.

Before, we apply these distinctions in the next section, we can define as follows:

Def. induction quality: A measure of the sensitivity of an empirical set-up (given two specified point-hypotheses and a fixed sample size) that is stated as $\alpha$ - and $\beta$-error. Though a set-up's acceptability rests on convention, equating both errors $(\alpha=\beta)$ avoids a bias pro detection ( $\alpha$-error) and con replicability ( $\beta$-error). Currently, $\alpha=\beta=0.05$ or $\alpha=\beta=0.01$ are common standards. Based on Neyman-Pearson theory, this measure is restricted to the discovery context; it qualifies the test-condition itself. Since we can gauge induction quality without actual data, this has nothing to do with a hypothesis test.

Def. corroboration quality: A comparative measure of the inductive support that data lend to hypotheses, stated as the likelihood-ratio (aka 'Bayes-factor') of two point-hypotheses given data of sufficient induction quality. The support threshold is the ratio $(1-\beta$-error $) / \alpha$-error, and so depends on induction quality of data. For instance, setting $\alpha=\beta=0.05$ yields a threshold of 19 (or $\log 19=1.28$ ), and $\alpha=\beta=0.01$ yields $99(\log 99=2.00)$, etc. Based on Wald's non-sequential testing theory the measure tests hypotheses against actual data in the justification context (Azzalini, 1996; Royall, 1997).

Of course, the final letter in NHST continues to abbreviate the term 'test.' After all, NHST does test the probability of data given a hypothesis, $P(\mathrm{D}, \mathrm{H})$. But our definitions imply that data of low replication probability are insufficient to test a hypothesis in the sense of gauging its likelihood, $L(\mathrm{H} \mid \mathrm{D})$. This is because a lowpowered "discovery" of effect $\mathrm{E}$ in test-condition $\mathrm{C}$ (as indicated by a large $\beta$-error) entails the improbability of redetecting $\mathrm{E}$ in subsequent instances of C. So even in view of a confirmatory likelihood-ratio, data of insufficient induction quality may wellinitially support a hypothesis. But similar support need not arise in new data of sufficient induction quality. So a given hypothesis may subsequently fail to be corroborated.

\section{Upshot}

On this background, the ongoing debates between statistical "schools" seem to be academic ones. After all, most extant estimation procedures for statistical significance operate squarely in the data space (Royall, 1997; Gelman, 2011; Wetzels et al., 2011). But if future theoretical developments must come to rely on coordinated activities that integrate the data with the hypotheses space, then the current crisis of empirical psychology would (at least partially) have arisen as a consequence of a methodologically unsound transition from discovery to justification. A sound version thereof, as we saw, leads from stable effects to trustworthy discoveries and on to acceptable forms of hypothesis corroboration.

As we also saw, integrating both spaces presupposes that we specify the expected empirical observation as a point-value. This states a theoretically sound minimum effect size (whether derived from a theory, or not); in uncertain cases it states a two-point interval placed around that value. By contrast, all alternative strategies simply let data have the "last word" on how we should construct a data-saving hypothesis. But this runs directly into the unmet challenge of validating induction.

Indeed, the risk of being "perfectly wrong" should be accepted even for precise theoretical assumptions. After all, being "broadly right" under merely vague assumptions is to accept virtually all non-random data-saving hypotheses. But that obviously fails to inform theoretical knowledge.

\section{CASE STUDY: PSI-RESEARCH}

\section{Overview}

To clarify the relation between hypothesis corroboration and data replication, we exemplify our distinctions with a fairly controversial effect, treat its size, point to future research needs, and summarize the main insight.

\section{Replicating Bem's Psi-hypothesis}

Bem's (2011) infamous results on precognition allegedly support the hypothesis that future expectations influence present behavior (aka 'psi-hypothesis'). Seeking to replicate Bem's data, Wagenmakers et al. (2012) claimed to pursue a confirmatory research agenda. They could stop inquiry after 200 sessions with 100 subjects (see their Figure 2; ibid., p. 636). For by then their data had lent 6.2 times more support to the $\mathrm{H}_{0}$ (read: $n o$ influence from future expectations) than to the $\mathrm{H}_{1}$ (read: influence). This is considered substantial evidence for the $\mathrm{H}_{0}$ (Jeffrey, 1961; Wagenmakers et al., 2011). Since Wagenmakers and colleagues base their inquiry on Wald's (1947) sequential analysis, however, we see reasons to treat their result with caution.

Rather than in order to evaluate theoretical hypotheses, Wald (1947) had developed sequential testing during WWII 
as a quality-control method in ammunition production. Its immediate purpose was to estimate how many shells in a lot deviate from a margin of error, $M$. While any deviation exceeding $M$ provides a sufficient reason to discard the whole lot, measuring large deviations from $\mathrm{M}$ is of course less cumbersome than measuring small ones. So it saves effort to infer probable but unobserved small deviations from large observed deviations.

Wald's sequential testing strategy provides a rather brilliant solution to the classical problem of inducing properties of the whole from its parts. But it cannot generalize to additional lots. Nor was it intended to induce over abstract categories, but rather over material objects. Applied to the case of Wagenmakers and colleagues testing the specified hypotheses $p=0.50$ vs. $p=0.531$ (based on Bem's 2011 first experiment), this means that neither the $\alpha$ - nor the $\beta$-error were known. After all, the number of observations keeps varying with the observed result. Without at least stipulating both errors, however, we cannot quantify the replicability of their result. So we should rather not trust it.

To explain, our previous section had shown that hypothesis testing requires trustworthy data. To more fully appreciate that trustworthiness is largely owed to knowledge of errors (Mayo, 1996, 2011), recall that induction quality and corroboration quality are related: if induction quality is unknown, then corroboration quality remains diffuse, and so can at best facilitate a vague form of justification. After all, even if a new and larger sample includes "old" data, a subsequent sample may nevertheless lead to a contrary decision as to whether a hypothesis is confirmed, or not.

As Rouder's (2014) discussion of the stopping rule shows (nicely), it is for this reason that Bayesians recommend that we keep adjusting our confidence level as data come in. Indeed, Bayesian inference puts the "focus on the [current] degree of belief for considered models [here: $\mathrm{H}_{1}$ and $\mathrm{H}_{0}$ ], which need not and should not be calibrated relative to some hypothetical truth" (ibid., p. 308). Hence, the authors could reject Bem's hypothesis $\left(\mathrm{H}_{1}\right)$, and instead accept the $\mathrm{H}_{0}$ at a confidence level of $(1-\alpha$-error $) / \beta$-error $=1.64$, where $1-\alpha=0.95$ and $\beta=0.58$.

But the matter is more intricate yet. After all, corroboration quality would change if we altered the presumed distribution of possible data (aka 'the priors'), for instance from a Cauchyto a normal-distribution (Bem et al., 2011). Consequently, the same actual data would now rather support the alternative hypothesis. In general, which hypothesis it is that data confirm can be manipulated-intentionally or not-by suitably selecting the distribution of possible data. So "that different priors result in different Bayes factors should [indeed] not come as a surprise" (Ly et al., 2016, p. 12).

The selected type of prior distribution, however, is logically independent of the hypothesis we wish to test, ever entails weighing one hypothesis against another, and ultimately reflects a subjective decision. This provides reasons against giving Bayesfactors alone the final say in hypothesis corroboration. By contrast, to point-specify the hypothesis one does test eliminates this caveat by avoiding the priors, and no other method does. In the absence of a specified $\mathrm{H}_{1}$, then, whether a test-condition suffices for a clear justification of the $\mathrm{H}_{0}$ depends on it featuring acceptably low $\alpha$ - and $\beta$-errors.
It follows that, had Wagenmakers and colleagues stopped their test after a mere 38 sessions, the same sequential testingstrategy should have led them to accept the $\mathrm{H}_{1}$ on the basis of nearly substantial evidence (a likelihood-ratio of 3 ). This would have "confirmed" Bem's result by replicating his data. (See the curve in Figure 2 of Wagenmakers et al., 2012, p. 636; Simmons et al., 2011 also illustrate this issue.) Given the small effect size $g=3.1 \%$ as a theoretical specification of prior results $(50 \%$ against $53.1 \%$, according to Bem, 2011, p. 409, experiment 1), one should therefore construct a sufficiently strong test-condition to obtain data of sufficient induction quality. ${ }^{6}$

Neyman-Pearson theory defines the necessary sample size (or number of observations, subjects, sessions, etc.) to firmly decide between two hypotheses with a difference of $g=3.1 \%$. Since this is a test against the $\mathrm{H}_{1}$, we should treat both errors equally, for instance by setting $\alpha=\beta=0.05$. It follows that, for the $\mathrm{H}_{0}$ to be accepted, it must be 19 times (0.95/0.05) more probable than the $\mathrm{H}_{1}$. The necessary sample size (for a proportion difference measured against a theoretical constant of 0.50) then comes to $n=2829$ (see Cohen, 1977, p. 169). Comparing this tall figure to the $n=200$ that Wagenmakers et al. (2012) report should make clear why one cannot trust their result.

A well-suited approximation of the necessary sample size, $\mathrm{n}$, given specified errors and a postulated effect size of mean differences, $\mathrm{d}$, is $\left(2\left(z_{(1-\alpha)}+z_{(1-\beta)}\right)^{2}\right) / \mathrm{d}^{2}=\mathrm{n}$, where $z_{(1-\alpha)}$ and $z_{(1-\beta)}$ increase provided $\alpha$ and $\beta$ decrease, with $z$ taking values greater than 1 , and $d$ mostly remaining below 1 . Given acceptable errors, a very small d (or g) thus generates a large n. So to achieve reasonable certainty under specified errors, we may incur an extremely large number of data points.

The main reason for the large $\mathrm{n}$ is the small difference between the two rivaling hypotheses and our rigor in controlling both errors. Therefore, it does not suffice to publish the testing-strategy before and a stopping-rule after data inspection (Nosek and Bar-Anan, 2012; Nosek et al., 2012, 2015). Similarly, though sequential testing is a less problematic way of inflating the $\alpha$-error than "double-dipping" (Kriegeskorte et al., 2009), it necessarily inflates the $\beta$-error, given that we hold effects constant. So it increases the chance of not detecting a true difference, making it improbable to replicate data in independent studies.

\section{Gauging the Psi-effect}

In the case of replicating the psi-hypothesis, the $\beta$-error was at least $\beta=0.58$, given $n=200, \alpha=0.05$ (one-sided), and $g=0.05$. Though this is a slightly higher value than was in fact observed, it still qualifies as a small effect (see Cohen, 1977, p. 155). In fact, G*Power software (Faul et al., 2007) estimates an even larger error of $\beta=0.64$. At any rate, the large $\beta$-error renders Wagenmakers and colleagues' test-condition unacceptable.

Based on extant psi-studies, Bem had formulated a $\mathrm{H}_{1}$ of $d=0.25$. (Bem prefers $t$-tests and more "classical" effect size measures over a binomial test; though results are stated in

${ }^{6} \mathrm{We}$ use ' $\mathrm{g}$ ' to express the effect size as the difference between a stipulated proportion of 0.50 and an observed proportion, using the sign test; ' $d$ ' denotes a difference between numerical means (Cohen, 1977). Notice that when errors and likelihoods are calculated via a normal curve approximation of the effect size, the model remains constant under different proof distributions. 
percentages, the output of both kinds of tests is equivalent.) Having specified d, we can thus calculate the error probabilities: for $\alpha=0.05$ (one-sided), $d=0.25$ and $n=100$, we find $\beta$-error $=0.18$. Now setting $\alpha=\beta=0.05$, the necessary sample is $n=175$, smaller than immediately above because $\mathrm{d}$ now is comparably large. ( $n=175$ is about half the sample the above formula approximates, since a difference between a constant and an empirical mean is evaluated. $)^{7}$ The Bayes-factor thus registers some 22 times in favor of a $\mathrm{H}_{1}$ postulating $d=0.25$. On a Bayesian view, this is a clear corroboration of the $\mathrm{H}_{1}$ over the $\mathrm{H}_{0}$.

Though the critical value $(1-\beta$-error $) / \alpha$-error $=0.80 /$ $0.05=16$ has now been surpassed, upon inspecting induction quality it transpires that the result is insufficiently stable. To explain, some of Bem's trials sought to induce arousal by displaying erotic and non-erotic pictures in random order, measured the degree of arousal before displaying a picture-type, and interpreted heightened arousal before showing an erotic picture as evidence of precognition. (This suffices to interpret the set-up as including a control group of sorts.) The observed effect was $d=0.19$ with $n=100$ in both samples (erotic vs. non-erotic). Comparing this with the hypotheses $d=0.00$ and $d=0.25$, however, a Bayes-factor of 2.18 is now too low. So Bem's first experiment indeed "discovers" a deviation from random (given $\alpha=0.05$, one-sided, and $1-\beta=0.82$ ). But the effect isn't stable (i.e., its reproducibility is insufficiently probable), particularly given that both hypotheses had been specified by recourse to meta-analytical results.

This goes to show that a Bayes-factor may well be extremely large although the effect is not trustworthy. Indeed, if we interpret data from the display of non-erotic pictures as a control groupas we should, because data from the display of erotic pictures did not significantly deviate from random-then a likelihood-ratio of 2.18 (i.e., a logarithm of 0.34 ) is hardly any evidence for a psieffect, even if we ignore its low replication probability. Rather, a firm decision under sufficient induction quality requires another sample of $n=75$ (see below).

\section{Meta-analyses of Additional Replication Attempts}

Bem's (2011) thought-provoking research has meanwhile initiated something like a research program. But we cannot elucidate its contradictory results by relying on either frequentist or Bayesian approaches to statistical inference. It should therefore be of interest to clarify the psi-debate by integrating both approaches.

Galak et al. (2012) and Bem et al. (2016) have conducted two independent meta-analyses of psi-studies. Their combination in fact yields the necessary sample size. The first study concludes negatively:

"Across seven experiments $(N=3,298)$, we replicate the procedure of experiments 8 and 9 from Bem (2011), which had originally demonstrated retroactive facilitation of recall.

\footnotetext{
${ }^{7}$ Here restricting the focus to a two sample (Neyman-Pearson) $t$-test with $\alpha$ - and $\beta$-errors, a draft on the one sample $t$-test and the negative consequences of various "saving-strategies" to reduce the sample size can be obtained from the authors.
}

We failed to replicate that finding. We further conduct a metaanalysis of all replication attempts of these experiments and find that the average effect size $(d=0.04)$ is not different from 0" (Galak et al., 2012, p. 933; italics added).

But the second meta-analysis arrives at a quite different result:

"We here report a meta-analysis of 90 experiments from 33 laboratories in 14 countries which yielded an overall effect greater than six sigma, $z=6.40, p=1.2 \times 10^{-10}$ with an effect size (Hedges' g) of 0.09. A Bayesian analysis yielded a Bayes Factor [BF] of $5.1 \times 10^{9}$, greatly exceeding the criterion value of 100 for 'decisive evidence' in support of the experimental hypothesis. When Bem's experiments are excluded the combined effect size for replications by independent investigators is $0.06, z=4.16, p=1.1 \times 10^{-5}$, and the $\mathrm{BF}$ value is 3.853 , again exceeding the criterion of 'decisive evidence.' [...] P-curve analysis, a recently introduced statistical technique, estimates the true effect size of the experiments to be 0.20 for the complete database and 0.24 for the independent replications" (Bem et al., 2016, p. 1).

To prepare for a critical discussion, consider that both meta-analyses sought to discover a non-random effect, but neither tested the psi-hypothesis in the sense of gauging $L(\mathrm{H} \mid \mathrm{D})$; effect sizes are heterogeneous, suggesting that uncontrolled influences are at play; Bem's own studies report larger effects than their independent replications, suggesting a self-fulfilling prophecy; Bayes-t-tests, as we saw, depend on the prior distribution and different priors can lead to contradictory results; most studies included in these meta-analyses are individually underpowered.

Of course, to simply aggregate various underpowered studies will not yield a trustworthy inductive basis. After all, almost all mean differences become statistically significant if we arbitrarily divide a sufficiently large sample $(n \geq 60.000)$ into two subgroups (Bakan, 1966). So provided that only the $\mathrm{H}_{0}$ is specified, we can almost always obtain a non-random result by increasing the sample. [This contrasts with the methodology of physics (Meehl, 1967), for instance, where a theoretical parameter is fixed and increasing the sample size eventually disproves a theory.]

In view of the more than 90 psi-studies that both metaanalyses reviewed, researchers did particularly consider the point-hypotheses $d=0.00$ (random, $\mathrm{H}_{0}$ ) and $d=0.25$ (specified, $\mathrm{H}_{1}$ ). (Bem assumed the latter $d$-value to plan studies with power $=0.80$, after "predicting" $d=0.24$ by analyzing independent replications; see Bem et al., 2016.) Since this "research program" requires amendment before it can successfully address the challenges the replicability crisis has made apparent, we now exemplify the inference strategy a genuine psi-research program would pursue.

Among the 90 psi-studies, we consider most trustworthy those that arose independently of Bem's research group, that are classified as exact replications, and that are peer-reviewed (These admittedly rigorous criteria leave but nine of the studies listed in Table A1 in Bem et al., 2016, p. 7, Dataset S1). 
Our first critical question must pertain to induction quality: given $\alpha=\beta=0.05$ and $d=0.25$ (see above), the necessary sample is $n=175$. The total sample from the nine studies comes to $n=520$. Since this far exceeds our requirements, we can in fact assess corroboration quality more severely. Given $\alpha=\beta$ and $n=520$, the critical value to corroborate the $\mathrm{H}_{1}$ now is $(1-\beta$-error $) / \alpha$-error $=0.998 / 0.002=499($ or $\log 2.70)$, and correspondingly for the $\mathrm{H}_{0}$, i.e., $(1-\alpha$-error $) / \beta$-error. Adding the log-likelihood-ratios for the $\mathrm{H}_{0}$ and subtracting those for the $\mathrm{H}_{1}$ then yields 5.13. This value is much higher than 2.70, making it 135,000 times more probable that data inductively support the $\mathrm{H}_{0}$, rather than a psi-effect of size $d=0.25$.

When we gauge the average effect size of our nine studies (weighed by the sample size), moreover, maximum likelihood estimation yields $d=0.07$ as a psi-effect that is 3.64 times more probable than a random effect. Rather than a final verdict on the true hypothesis, of course, this provides but a relative corroboration of one hypothesis against another. A maximum likelihood estimate that registers so close to the hypothetical parameter, however, fails to provide any hint for further research.

The $\mathrm{H}_{0}$ is now much better corroborated than the psihypothesis. Of course, this does not falsify a psi-hypothesis postulating a yet smaller effect. It is nevertheless reasonable to reject the psi hypothesis. After all, to theoretically explain an effect only becomes more difficult the smaller it is. But again, one cannot be certain. ${ }^{8}$

\section{Summary}

Our discussion shows that a firm and transparent decision between two specified hypotheses requires knowing the effect size and selecting tolerable $\alpha$ - and $\beta$-errors. We also saw how this determines the necessary sample size. Specifically, the smaller an effect is the larger is the necessary sample. Theories that predict small effects should therefore be confronted with much larger samples than is typical. Conversely, the small effects and samples that top-tier journals often report let few published effects count as probably replicable. Indeed, the average test-power reported in Bakker et al. (2012) "predicts" the lower bound (36\%) of the average replication rate reported by Open Science Collaboration (2015).

Since many studies avoid specifying the alternative hypothesis, researchers may at best hope to find a significant effect among their results. This partially explains why they sometimes "torture" data until significance is achieved. As we saw, such results frequently surface as allegedly important effects, that is, as genuine discoveries (see Fanelli and Glänzel, 2013; Witte and Strohmeier, 2013). Predictably, top-tier journals regularly

\footnotetext{
${ }^{8}$ It is natural to object that very few people may in fact command psi-abilities. This might seem to explain why Bem can measure only a small effect. However, this explanation-sketch presupposes that we could (somehow) aggregate effect sizes from individually underpowered studies into a "pooled" effect size. Instead, what we can safely aggregate are log-likelihoods (see Witte and Zenker, 2016a,b, 2017). The explanation-sketch might nevertheless lead to a new hypothesis, namely: can we reliably separate subjects into those who command and those who lack psiabilities? If so, we should next test among the former group if the psi-effect is stable. At any rate, research addressing such hypotheses qualifies as a discovery context activity.
}

publish studies that fail to report probably replicable discoveries, namely when their test-power is too low $(<0.80$ or $<0.95)$ to safely reject the $\mathrm{H}_{0}$ (Bakker et al., 2012; Francis, 2012; Open Science Collaboration, 2015; Etz and Vandekerckhove, 2016).

With these insufficiently designed studies as evidence for a goal-conflict between psychologists and their field (Witte, 2005; Eriksson and Simpson, 2013), we go on to show how research programs improve the status quo.

\section{RESEARCH PROGRAMS}

\section{Four Developmental Steps}

This section outlines the four steps a progressive research program takes in order to improve empirical knowledge (Hacking, 1978; Lakatos, 1978; Larvor, 1998; Motterlini, 2002). Adopting such programs is a natural consequence of recognizing the precisification of hypotheses as a necessary condition to obtain trustworthy results, and of accepting that prior (fallible) knowledge informs future research.

\section{Step One: Ideas without Controlled Observation}

The first step involves an idea or intuition, perhaps acquired in what C.S. Peirce called retroduction. Interesting in itself, having that intuition is less relevant for developing a scientific construct. Though an intuition is by definition not based on conscious observation, to be further explored it must nonetheless sufficiently impress us. For instance, we might seek hints in subjective experience, theoretical observation, or collegial discussion. Once we are convinced that the idea is relevant, we can engage with it systematically, perhaps moving from material at hand to thought-experiments, computer simulations, or an "idea-paper" (sans significance tests, etc.). Importantly, such activities are possible without collecting data.

\section{Step Two: Devising an Empirical Set-up}

The second step establishes our idea with a method, so that it may potentially count as a genuine discovery. This requires controlling an empirical set-up in order to "observe" the phenomenon. But we saw that observations may mislead because of random effects and sampling- or measurement-error. So for a discovery to be established, the phenomenon must significantly deviate from random. After all, random variation lacks specific semantic content and so does not explain anything but itself.

We also saw that proofs under probabilistic variation are based on inferential statistics that consider the observation's $p$-value given the $\mathrm{H}_{0}$. The classical claim ascribed to Fisher is that a small $p$-value reflects a rare enough event in a random model. (At this step, we cannot properly call the search for small $p$-values ' $\mathrm{p}$-hacking, an often observed strategy after having obtained data.) As a consequence, a "large p" $(p>0.05)$ signals our failure to measure a significant deviation from random. 
This prima facie suggests complicity with a random model. But a large $p$ is expected, of course, if the set-up is insufficiently sensitive to detect an effect that nevertheless is present. So it would be naïve to treat the absence of a statistically significant deviation from random as conclusive evidence for the effect's absence.

This sensitivity caveat calls on us to adjust our logic of decision-making. After all, statistically insignificant deviations from random may still signal epistemic value. Historically, that insight led Neyman-Pearson-test-theory to provide us also with an estimate of the $\beta$-error. This should be sufficiently small for the chance of not detecting a true effect to be low. In other words, the set-up's sensitivity should be sufficiently high.

A set-up will generally be optimal if evidence features the smallest possible $p$-value given the random model, on one hand, and the largest probability of registering each true deviation from random using a minimum number of observations, on the other. Moreover, an optimal test should be unbiased, so that we can almost certainly detect a true effect as we increase the number of observations. In short, we should select the most powerful test-condition. Test-power considerations thus inform how we gauge the relative inductive support that data provide for a content-free $\mathrm{H}_{0}$, compared to the support that the same data provided for a (one- or two-sided) $\mathrm{H}_{1}$ of substantial content.

At this point, however, rather than manage a pointhypothesis, we still deal with a vague $\mathrm{H}_{1}$. Moreover, we know induction quality of data but partially as long as we merely hold the $\alpha$-error constant, but not the $\beta$-error. As a consequence, data may now seem to corroborate or falsify a hypothesis, but they may not be replicable. Lastly, corroboration and falsification equally depend on the distribution of possible data. So corroboration quality is at best diffuse.

Even if data should now "eliminate" the $\mathrm{H}_{0}$, as it were, a genuine bundle of substantial alternative $\mathrm{H}_{1}$-hypotheses remains to be eliminated. Since each such $\mathrm{H}_{1}$-hypothesis postulates a distinct effect size, the whole bundle provides mutually exclusive explanatory candidates. Each such $\mathrm{H}_{1}$ therefore associates to a distinct likelihood-ratio as its corroboration measure. So the unsolved problem of validating induction merely let data hint at the correct theoretical parameter, but data alone cannot determine it. Instead, it is our having further specified this parameter that eventually lets data decide firmly between any two such point-hypotheses.

\section{Step Three: Replication and Meta-analysis}

As we achieve replication-success several times, we can give a better size-estimate of a significant effect. But this estimate will be unbiased only if we also base it on (often unpublished) statistically non-significant results (Sterling, 1959; Scargle, 2000; Schonemann and Scargle, 2008; Ferguson and Heene, 2012). Moreover, an effect size that has remained heterogeneous across several studies should eventually be differentiated from its test-condition. In fact, this is the genuine purpose of a meta-analysis. But extant analyses are heavily biased toward published results. Despite various bias detection-tools, such analyses therefore often present a skewed picture of all available data, and hence exaggerate the true effect size (Francis, 2012).

When we reproduce an effect, we should therefore correct a plain induction over prior findings by more theoretical strategies (see, e.g., Witte, 1994, 1996b, 2005). This includes data-inspection and -reconstruction by means of a theory with a mathematical core that predicts quantitative results while retaining an adequate connection to data. Data reconstruction thus becomes a stepping stone to formulating point-hypotheses.

The forgoing exhausts the relevant discovery context activities. Subsequent work should be guided by specifying effect sizes as point-hypotheses, and by testing them against new data that arise as retrodictions or predictions.

\section{Step Four: Precisification of Effect Sizes and Theoretical Construction}

As we saw, we can quantitatively assess the quality of an empirical set-up only after a point-hypothesis is available. Subsequently, a set-up's induction quality can serve as a criterion to probabilistically corroborate, or falsify, a theoretical construct. So the fourth step directly concerns formulating precise pointhypotheses.

A clear indicator that we have reached the justification context is to inspect likelihoods of hypotheses given data, $L(\mathrm{H} \mid \mathrm{D})$, rather than probabilities of data given hypotheses, $P(D, H)$. Whether precision-gains then arise from a parameter-estimation or by combining significant results (Witte and Zenker, 2016a,b, 2017), here we either induce over empirical results, or we perform a quantitative reanalysis. This marks the onset of an explanatory construction (Witte, 1996b; Witte and Heitkamp, 2006). As a rule, if we have corroborated an effect size, we should next provide a theoretical (semantic) explanation.

Generating such explanations takes time, of course, and often incurs unforeseen problems. Indeed, it need not succeed. With Lakatos (1978), we consider a research program progressive as long as a theoretical construction or its core-preserving modification generate predictions that are at least partially corroborated by new data of sufficient induction quality. Any such construction may therefore lead to further discoveries, e.g., in the form of observations that deviate from random. So it remains overly simple to treat theory-development as a linear process (see Figure 1). But that scientific progress grounds in fallible knowledge of an original phenomenon deserves acceptance.

A basic principle is to continue the precisification of hypotheses, since this improves both induction quality (from unknown to known to be probably reproducible) as well as corroboration quality (from diffuse to precise). A progressive research program thus shifts the focus, away from vague justification against the $\mathrm{H}_{0}$, toward precise justification of a point-specified $\mathrm{H}_{1}$. That said, HARKing and double dipping (Kerr, 1998; Kriegeskorte et al., 2009; Simmons et al., 2011) 


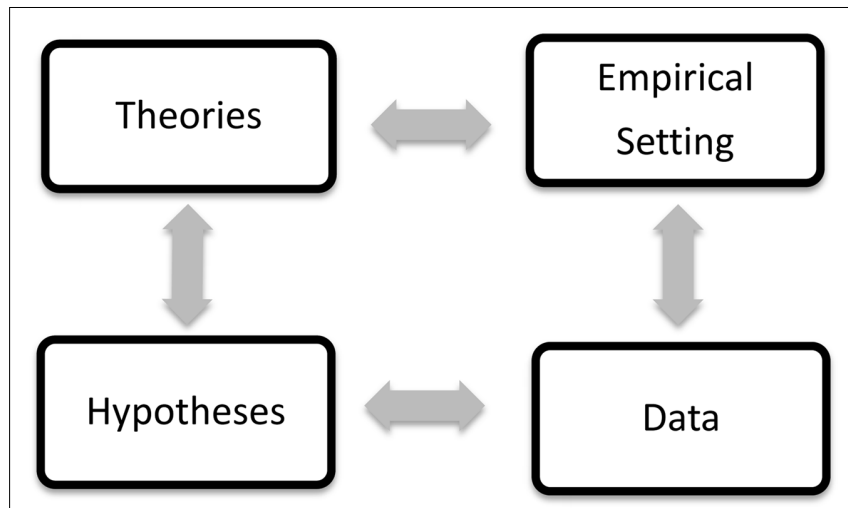

FIGURE 1 | Only stable data ("phenomena") can be meaningfully subsumed under empirical hypotheses (bottom arrow); such hypotheses can be systematized into theories (left arrow) that retrodict old and predict new data; this may lead to constructing empirical set-ups (top arrow) for new phenomena (right arrow), which potentially improve extant theories.

remain non-cogent justifications because each so generated result must lead to a confirmation.

\section{MOVE OVER, PLEASE!}

Statistical inference is a tool to corroborate theoretical assumptions rather than a machinery to generate theoretically grounded empirical knowledge-which an empirical science must justify with trustworthy data. Therefore, a loose discovery under vague hypotheses (based on sample-estimations) can never lead to a firmly corroborated theoretical assumption. In fact, to even only disconfirm a precise assumption is already far more informative than to never achieve adequate precision.

Considering the scarcity of similarly well-hardened knowledge in current psychological research, the field's progress demands justification context-activities (Ioannidis, 2012). Indeed, as long as the field as a whole remains in the discovery context, it is doubtful that lenient reviewers, full data disclosure, or removing the publication-bottleneck, etc. even address the true challenges. Instead, we would presumably see metaanalyses lead to meta-meta-analyses, which however cannot serve a progressive research program (Schmidt et al., 2009; Cafri et al., 2010; Stegenga, 2011; Chan and Arvey, 2012; Ferguson and Heene, 2012; Mitchell, 2012). Rather, if a metaanalysis discovers an effect, one should seek to confirm a precisified version thereof by successfully predicting it in new data. $^{9}$

The likelihood ratio, as we saw, is the measure of corroboration quality. For a tolerable error-range, reasonable certainty that a $\mathrm{H}_{1}$ is justified thus requires that its likelihoodratio exceeds a conventional threshold. We can compare the error terms from Neyman-Pearson-theory to Jeffrey's (1961)

\footnotetext{
${ }^{9}$ Such research clearly differs from parameter-estimation because it can bury "undead" theories. But this is impossible given how standard methods are mostly used today (Ferguson and Heene, 2012; Eriksson and Simpson, 2013).
}

qualitative classification: $\alpha=\beta=0.05$ translates into a likelihoodratio of 19 ("strong evidence") and $\alpha=\beta=0.01$ into a ratio of 99 ("very strong evidence," "nearly extreme evidence"). Further, provided that $\alpha=\beta \leq 0.01$, the degree of corroboration for the supported hypothesis will in the long run approach the test-power value (Wald, 1947).

As a summary, we now list six increasingly important results of a research program and their measures. The last result corroborates a point-hypothesis.

(1) preliminary discovery: $\alpha$-error or merely a $p$-value (to establish that data are non-random)

(2) substantial discovery: $\alpha$ and $1-\beta$-error (to gauge replicability based on a specific effect size and a particular sample size)

(3) preliminary falsification of the $\mathrm{H}_{0}: L\left(\mathrm{~d}_{\mathrm{H} 1}>0\right) / L\left(\mathrm{~d}_{\mathrm{H} 0}=0\right)$ (to establish a $\mathrm{H}_{1}$ that deviates in one direction from the $\mathrm{H}_{0}$ as more likely than the random parameter $d=0$ )

(4) substantial falsification of the $\mathrm{H}_{0}: L\left(\mathrm{~d}_{\mathrm{H} 1}>\Delta\right) / L\left(\mathrm{~d}_{\mathrm{H} 0}=0\right)$, where $\Delta$ is the theoretical minimum effect size value (to establish a $\mathrm{H}_{1}$ as non-random and also exceeding $\Delta$ )

(5) preliminary verification of the $\mathrm{H}_{1}: L\left(\mathrm{~d}_{\mathrm{H} 1}=\Delta\right) / L\left(\mathrm{~d}_{\mathrm{H} 0}=0\right)$ (to corroborate the theoretical parameter $\Delta$ against the random parameter)

(6) substantial verification of the $\mathrm{H}_{1}$ : $L\left(\mathrm{~d}_{\mathrm{emp}}\right.$, $\left.\mathrm{H}_{1}\right) / \mathrm{L}\left(\mathrm{d}_{\mathrm{H} 2}=\Delta\right)<4$, given approximately normally distributed data, where $\mathrm{d}_{\mathrm{emp}}$ is the empirical effect size ${ }^{10}$ (to indirectly corroborate $\Delta$ against the maximum-likelihood estimate of empirical data, $\mathrm{d}_{\mathrm{emp}}$ ).

The justification context starts with line (3). So the unsophisticated application of NHST sees large parts of empirical psychological research "stuck" with making preliminary or substantial discoveries. Notice, too, that the laudable proposal by Benjamin et al. (2017) to drastically lower the $\alpha$-error merely addresses what shall count as a preliminary discovery. But it leaves unaddressed how we establish a substantial discovery as well as the justification context as a whole.

\section{PRECISE THEORETICAL CONSTRUCTS?}

Allow us to briefly speculate why the goal conflict between generating statistically significant results and generating trustworthy fallible knowledge has played out in favor of the former goal. We saw that trustworthy data is primarily relevant toward developing and testing more refined theoretical constructs. But theory-construction is rarely taught at universities (Gigerenzer, 2010). So the replicability crisis also showcases our inability to in fact erect the constructs that statistically significant effects should lead to (Ellemers, 2013; Klein, 2014).

To give but two examples, the 51 theories collected in a recent social psychology handbook (van Lange et al., 2012)

${ }^{10}$ If the likelihood value of the theoretical parameter does not fall outside of the 95\%-interval placed around the maximum-likelihood-estimate, then we view the theoretical parameter as substantially verified, and thus corroborated. After all, both the empirical result and the theoretical assumption now lie within an acceptable interval. The corroboration threshold is given by the ratio of the two likelihood-values, i.e., the maximum ordinate of the normal curve (0.3989) and the ordinate at the 95\%-interval (0.10). To good approximation, this yields 4 . 


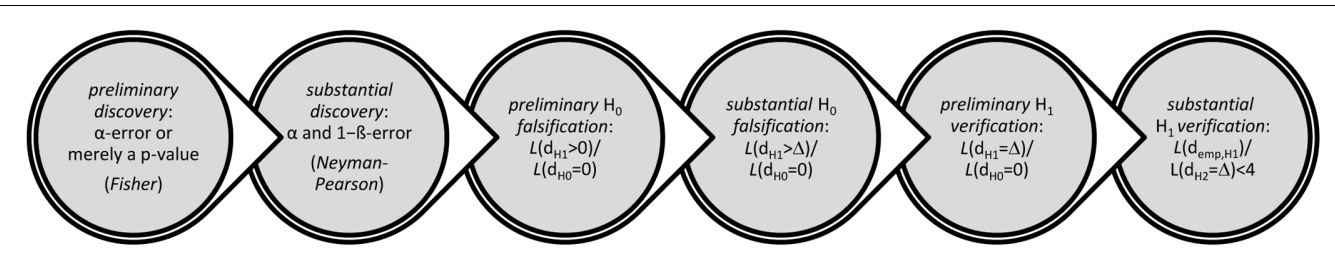

FIGURE 2 | Salient results of a research program.

cannot achieve equally precise predictions as those that a random model offers. But not only should we base a fair decision between two hypotheses on data that are known to be probably replicable; we should also require parity of precision (Witte and Kaufman, 1997). By contrast, a two volume edition on small group behavior (Witte and Davis, 1996) contains eleven theories that are sufficiently elaborated to predict precise effects, while another ten theories make vague predictions. So precise constructions are possible, yet they are rare. Therefore, calling the replicability crisis "home-made" does not directly implicate career aspiration or resource shortage. Rather, ignorance of how one constructs a theory seems to be an important intermediate factor.

The crisis will probably seem insurmountable to those who disbelieve that a developing research program is even possible. Alas, might one not first ask to show us a failed research program? Indeed, would not a research program culture need to have been established, and to have broadly failed, too? If so, then an urgent challenge is to coordinate our research away from the individualistically organized but statistically underpowered short-term efforts that have produced the crises, toward jointly managed and well-powered long-term research programs.

Of course, it takes more than writing papers. Indeed, funding-, career- and incentive-structures may also need to change, including how empirical psychologists understand their field. It may even require that larger groups break with what most do quasi-habitually. This, perhaps, would establish what our paper only described.

\section{CONCLUSION}

The replicability crisis in psychology is in large part a consequence of applying an unsophisticated version of NHST. This praxis merely generates theoretically disconnected "oneoff" discoveries-that is, effects which deviate statistically significantly from a random model. Parallel to it runs the interpretative or rhetorical praxis of publishing such effects as scientifically important results, rather than as the parameter estimations they are. The former praxis fails to maximize the utility of a sophisticated version of NHST, which nevertheless remains a useful and elegant approach to gauging $P(\mathrm{D}, \mathrm{H})$. The latter praxis regularly over-reports $P(\mathrm{D}, \mathrm{H})$ as $L(\mathrm{H} \mid \mathrm{D})$, which even the most sophisticated application of NHST, however, cannot warrant. Both praxes may plausibly have arisen from not fully understanding the limits of NHST.
The field has thus "discovered" many small effects. But whenever empirical studies remain underpowered, because they comprise too few data-points, research efforts remain in the discovery context. Here, vague effect sizes and vague alternative hypotheses are normal. But such results fail to convince as trustworthy effects that deserve theoretical explanation. By contrast, a clear indicator that research has shifted to the justification context is a likelihood-ratio-based decision regarding a precisified effect size that is expectable in new data of known induction quality (entailing a Bayes-factor for fixed hypotheses).

This requires a diachronic notion of the research processa developing research program - that adapts statistical inference methods to prior knowledge. Schematically (Figure 2), we start with $p$-values (Fisher), move on to an optimal test against a random-model (Neyman-Pearson with $\alpha$ - and $1-\beta$-error), accompanied by parameter estimation via meta-analysis, to achieve-entering the justification context - a corroboration of a theoretically specified effect size based on stable data of known induction quality (under tolerable errors), against a random model or against another point-specified hypothesis.

In particular, we should deduce from a theory a specified effect size that goes beyond the simple assumption of a minimal effect. A quantitative specification without theoretical explanation may well be a first step (assuming known induction quality and precise corroboration quality). But a successful research program must derive a prediction from a theoretical model and provide an explanation of the effect's magnitude. For instance, psi-research would only gain from such an explanation.

Since many theories only offer vague predictions, moreover, the lack of confidence among psychologists might at least partially result from failed theory-development. But the replicability crisis itself is narrowly owed to underpowered studies. Making headway takes researchers who join forces and resources, who coordinate research efforts under a long-term perspective, and who adapt statistical inference methods to prior knowledge. To this end, we have also seen a strategy for combining data from various studies that avoids the pitfalls of extant meta-analyses. It is in the integrated long run, then, that empirical psychology may improve.

\section{AUTHOR CONTRIBUTIONS}

Both authors have jointly drafted this manuscript; the conceptual part of this work originates with EW; FZ supplied additional explanatory material and edited the manuscript. 


\section{ACKNOWLEDGMENTS}

For valuable comments that served to improve earlier versions of this manuscript, we thank Peter Killeen and Moritz Heene as well as $\mathrm{EB}$ and $\mathrm{PB}$ for their reviews. Thanks also to HF for overseeing the peer review process. All values were calculated using G*Power, V3.1.9.2 (Faul et al., 2007). FZ acknowledges funding

\section{REFERENCES}

Azzalini, A. (1996). Statistical Inference. Based on the Likelihood. London: Chapman \& Hall.

Bakan, D. (1966). The test of significance in psychological research. Psychol. Bull. 66, 423-437. doi: 10.1037/h0020412

Baker, M. (2015). First results from psychology's largest reproducibility test. Nat. News. doi: 10.1038/nature.2015.17433

Bakker, M., van Dijk, A., and Wicherts, J. M. (2012). The rules of the game called psychological science. Perspect. Psychol. Sci. 7, 543-554. doi: 10.1177/ 1745691612459060

Bem, D., Tressoldi, P., Rabeyron, T. H., and Duggan, M. (2016). Feeling the future: a meta-analysis of 90 experiments on the anticipation of random future events [version 2; referees: 2 approved]. F1000 Res. 4, 1188. doi: 10.12688/ f1000research.7177.2

Bem, D. J. (2011). Feeling the future: experimental evidence for anomalous retroactive influences on cognition and affect. J. Pers. Soc. Psychol. 100, 407-425. doi: $10.1037 / \mathrm{a} 0021524$

Bem, D. J., Utts, J., and Johnson, W. O. (2011). Reply. Must psychologists change the way they analyze their data? J. Pers. Soc. Psychol. 101, 716-719. doi: 10.1037/ a0024777

Benjamin, D. J., Berger, J., Johannesson, M., Nosek, B. A., Wagenmakers, E. -J., Berk, R., et al. (2017). Redefine Statistical Significance. Available at: psyarxiv.com/mky9j

Cafri, G., Kromrey, J. D., and Brannick, M. T. (2010). A meta-meta-analysis: empirical review of statistical power, type I error rates, effect sizes, and model selection of meta-analyses published in psychology. Multivariate Behav. Res. 45, 239-270. doi: 10.1080/00273171003680187

Chan, M.-L. E., and Arvey, R. D. (2012). Meta-analysis and the development of knowledge. Perspect. Psychol. Sci. 7, 79-92. doi: 10.1177/1745691611429355

Cohen, J. (1962). The statistical power analysis for the behavioral sciences: a review. J. Abnorm. Soc. Psychol. 65, 145-153.

Cohen, J. (1977). Statistical Power Analysis for the Behavioral Sciences (Rev. ed.). London: Academic Press.

Cowles, M. (1989). Statistics in Psychology. An Historical Perspective. Hillsdale: Erlbaum.

Ellemers, N. (2013). Connecting the dots: mobilizing theory to reveal the big picture in social psychology (and why we should do this). Eur. J. Soc. Psychol. 43, 1-8. doi: $10.1002 /$ ejsp. 1932

Eriksson, K., and Simpson, B. (2013). Editorial decisions may perpetuate belief in invalid research findings. PLOS ONE 8:e73364. doi: 10.1371/journal.pone. 0073364

Etz, A., and Vandekerckhove, J. (2016). A Bayesian perspective on the reproducibility project: psychology. PLOS ONE 11:0149794. doi: 10.1371/ journal.pone.0149794

Fanelli, D., and Glänzel, W. (2013). Bibliometric evidence for a hierarchy of the sciences. PLOS ONE 8:e66938. doi: 10.1371/journal.pone.0066938

Faul, F., Erdfelder, E., Lang, A.-G., and Buchner, A. (2007). G*Power 3: a flexible statistical power analysis program for the social, behavioral, and biomedical sciences. Behav. Res. Methods 39, 175-191. doi: 10.3758/BF03193146

Ferguson, C. J., and Heene, M. (2012). A vast graveyard of undead theories: publication bias and psychological science's aversion to the null. Perspect. Psychol. Sci. 7, 555-561. doi: 10.1177/1745691612459059

Fisher, R. A. (1956). Statistical Methods and Scientific Inference. New York, NY: Hafner.

Francis, G. (2012). The psychology of replication and replication in psychology. Perspect. Psychol. Sci. 7, 585-594. doi: 10.1177/1745691612459520 from the Ragnar Söderberg Foundation, an "Understanding China"-Fellowship from the Confucius Institute (HANBAN), as well as funding through the European Union's FP 7 framework program (No. 1225/02/03) and the Volkswagen Foundation (No. 90/531). Finally, thanks to Frontiers for a partial fee waiver, and to Lund University's Library and the Department of Philosophy for providing open access funding.

Fuchs, H. M., Jenny, M., and Fiedler, S. (2012). Psychologists are open to change, yet wary of rules. Perspect. Psychol. Sci. 7, 639-642. doi: 10.1177/1745691612459521

Galak, J., LeBoeuf, R. A., Nelson, L. D., and Simmons, J. P. (2012). Correcting the past: failures to replicate Psi. J. Pers. Soc. Psychol. 103, 933-948. doi: 10.1037/ a0029709

Gelman, A. (2011). Induction and deduction in Bayesian data analysis. Ration. Mark. Morals 2, 67-78.

Gigerenzer, G. (2010). Personal reflections on theory and psychology. Theory Psychol. 20, 733-743. doi: 10.1177/0959354310378184

Gilbert, D. T., King, G., Pettigrew, S., and Wilson, T. D. (2016). Comment on "Estimating the reproducibility of psychological science. Science 351, 1037. doi: $10.1126 /$ science.aad 7243

Hacking, I. (1978). Imre Lakatos's philosophy of science. Br. J. Philos. Sci. 30, 381-410. doi: 10.1093/bjps/30.4.381

Harlow, L. L., Mulaik, S. A., and Steiger, J. H. (eds) (1997). What If There Were No Significance Tests? Mahwah: Erlbaum.

Holcombe, A. O. (2016). Introduction to a registered replication report on ego depletion. Perspect. Psychol. Sci. 11, 545. doi: 10.1177/1745691616652871

Hoyningen-Huene, P. (2006). "Context of discovery vs. context of justification and Thomas Kuhn," in Revisiting Discovery and Justification, eds J. Schickore and F. Steinle (Dordrecht: Springer), 119-131.

Ioannidis, J. P. A. (2012). Why science is not necessarily self-correcting. Perspect. Psychol. Sci. 7, 645-654. doi: 10.1177/1745691612464056

Ioannidis, J. P. A. (2014). How to make more published research true. PLOS Med. 11:e1001747. doi: 10.1371/journal.pmed.1001747

Jeffrey, H. (1961). The Theory of Probability. Oxford: Oxford University Press.

Kerr, N. L. (1998). HARKing: hypothesizing after the results are known. Pers. Soc. Psychol. Rev. 2, 196-217. doi: 10.1207/s15327957pspr0203_4

Klein, S. B. (2014). What can recent replication failures tell us about the theoretical commitments of psychology? Theory Psychol. 24, 326-338. doi: 10.1177/ 0959354314529616

Kriegeskorte, N., Simmons, W. K., Bellgowan, P. S. F., and Baker, C. I. (2009). Circular analysis in systems neuroscience: the dangers of double dipping. Nat. Neurosci. 12, 535-540. doi: 10.1038/nn.2303

Kuhn, T. S. (1970). The Structure of Scientific Revolutions, 2nd Edn. Chicago: University of Chicago Press.

Lakatos, I. (1978). The Methodology of Scientific Research Programs. Cambridge: Cambridge University Press.

Larvor, B. (1998). Lakatos: An Introduction. London: Routledge.

Ly, A., Verhagen, J., and Wagenmakers, E.-J. (2016). Harold Jeffrey's default Bayes factor hypothesis tests: explanation, extension, and application in psychology. J. Math. Psychol. 72, 19-32. doi: 10.1016/j.jmp.2015.06.004

Maxwell, S. E. (2004). The persistence of underpowered studies in psychological research: causes, consequences, and remedies. Psychol. Methods 9, 147-163. doi: 10.1037/1082-989X.9.2.147

Mayo, D. G. (1996). Error and the Growth of Experimental Knowledge. Chicago: University of Chicago Press. doi: 10.7208/chicago/9780226511993.001.0001

Mayo, D. G. (2011). Statistical science and philosophy of science: where do/should they meet in 2011 (and beyond)? Ration. Mark. Morals 2, 79-102.

Meehl, P. E. (1967). Theory testing in psychology and physics: a methodological paradox. Philos. Sci. 34, 103-115. doi: 10.1086/288135

Mitchell, G. (2012). Revisiting truth or triviality: the external validity of research in the psychological laboratory. Perspect. Psychol. Sci. 7, 109-117. doi: 10.1177/ 1745691611432343

Motterlini, M. (2002). Reconstructing Lakatos: a reassessment of Lakatos' epistemological project in the light of the Lakatos Archive. Stud. Hist. Philos. Sci. 33, 487-509. doi: 10.1016/S0039-3681(02)00024-9 
Nosek, B. A., Alter, G., Banks, G. C., Borsboom, D., Bowman, S. D., Breckler, S. J., et al. (2015). Promoting an open research culture. Science 348, 1422-1425. doi: 10.1126/science.aab2374

Nosek, B. A., and Bar-Anan, Y. (2012). Scientific utopia: I. Opening scientific communication. Psychol. Inq. 23, 217-243. doi: 10.1080/1047840X.2012.692215

Nosek, B. A., Spies, J. R., and Motyl, M. (2012). Scientific utopia: II. Restructuring incentives and practices to promote truth over publishability. Perspect. Psychol. Sci. 7, 615-631. doi: 10.1177/1745691612459058

Open Science Collaboration. (2015). Estimating the reproducibility of psychological science. Science 349:acc4716. doi: 10.1126/science.aac4716

Pashler, H., and Wagenmakers, E.-J. (2012). Editors' introduction to the special section on replicability in psychological science: a crisis of confidence? Perspect. Psychol. Sci. 7, 528-530. doi: 10.1177/1745691612465253

Reichenbach, H. (1938). Experience and Prediction. Chicago: The University of Chicago Press.

Rosnow, R., and Rosenthal, R. (1989). Statistical procedures and the justification of knowledge in psychological science. Am. Psychol. 44, 1276-1284. doi: 10.1037/ 0003-066X.44.10.1276

Rouder, J. N. (2014). Optional stopping: no problem for Bayesians. Psychon. Bull. Rev. 21, 301-308. doi: 10.3758/s13423-014-0595-4

Rouder, J. N., Speckman, P. L., Sun, D., Morey, R. D., and Iverson, G. (2009). Baysian t-tests for accepting and rejecting the null hypothesis. Psychon. Bull. Rev. 16, 225-237. doi: 10.3758/PBR.16.2.225

Royall, R. (1997). Statistical Evidence. A Likelihood Paradigm. London: Chapman \& Hall.

Scargle, J. D. (2000). Publication bias: the "file-drawer" problem in scientific inference. J. Sci. Explor. 14, 91-106.

Schickore, J., and Steinle, F. (eds) (2006). Revisiting Discovery and Justification: Historical and Philosophical Perspectives on the Contest Distinction. Dordrecht: Springer.

Schmidt, F. L., Oh, I., and Hayes, T. L. (2009). Fixed versus random-effect models in meta-analysis: model properties and an empirical comparison of differences in results. Br. J. Math. Stat. Psychol. 62, 97-128. doi: 10.1348/000711007X255327

Schonemann, P. H., and Scargle, J. D. (2008). A generalized publication bias model. Chin. J. Psychol. 50, 21-29.

Sedlmeier, P., and Gigerenzer, G. (1989). Do studies of statistical power have an effect on the power of studies? Psychol. Bull. 105, 309-316. doi: 10.1037/00332909.105.2.309

Simmons, J. P., Nelson, L. D., and Simonsohn, U. (2011). False-positive psychology. Psychol. Sci. 22, 1359-1366. doi: 10.1177/0956797611417632

Spellman, B. A. (2012). Introduction to the special section on research practices. Perspect. Psychol. Sci. 7, 655-656. doi: 10.1177/1745691612465075

Stegenga, J. (2011). Is meta-analysis the platinum standard of evidence? Stud. Hist. Philos. Biol. Sci. 42, 497-507. doi: 10.1016/j.shpsc.2011.07.003

Sterling, T. D. (1959). Publication decisions and their possible effects on inferences drawn from tests of significance-or vice versa. J. Am. Stat. Assoc. 54, 30-34.

Sturm, T. H., and Mülberger, A. (2012). Crisis discussions in psychology: new historical and philosophical perspectives. Stud. Hist. Philos. Biol. Sci. 43, 425-433. doi: 10.1016/j.shpsc.2011.11.001

van Lange, P. A. M., Kruglanski, A. W., and Higgins, E. T. (2012). Handbook of Theories of Social Psychology, Vol. 1+2. London: Sage Publications.

Verhagen, A. J., and Wagenmakers, E.-J. (2014). Bayesian tests to quantify the result of a replication attempt. J. Exp. Psychol. Gen. 143, 1457-1475. doi: $10.1037 / \mathrm{a} 0036731$

Wagenmakers, E.-J., Wetzels, R., Borsboom, D., and van der Maas, H. L. J. (2011). Why psychologists must change the way they analyze their data: the psi case: comment on Bem (2011). J. Pers. Soc. Psychol. 100, 426-432. doi: 10.1037/ a0022790

Wagenmakers, E.-J., Wetzels, R., Borsboom, D., van der Maas, H. L. J., and Kievit, R. A. (2012). An agenda for purely confirmatory research. Perspect. Psychol. Sci. 7, 632-638. doi: 10.1177/1745691612463078
Wald, A. (1947). Sequential Analysis. New York: Wiley.

Wetzels, R., Matzke, D., Lee, M. D., Rouder, J. N., Iverson, G. J., and Wagenmakers, E.-J. (2011). Statistical evidence in experimental psychology: an empirical comparison using 855 t-tests. Perspect. Psychol. Sci. 6, 291-298. doi: 10.1177/ 1745691611406923

Willy, R. (1889). Die Krisis in der Psychologie. Leipzig: Reisland.

Witte, E. H. (1980). Signifikanztest und statistische Inferenz. Analysen, Probleme, Alternativen [Significance test and statistical inference: Analyses, problems, alternatives]. Stuttgart: Enke.

Witte, E. H. (1994). "Minority influences and innovations: the search for an integrated explanation of psychological and sociological models," in Minority Influence, eds S. Moscovici, A. Mucchi-Faina, and A. Maass (Chicago: NelsonHall), 67-93.

Witte, E. H. (1996a). "Small-group research and the crisis of social psychology: an introduction," in Understanding Group Behavior, Vol. 2, eds E. H. Witte and J. H. Davis (Mahwah: Erlbaum), 1-8.

Witte, E. H. (1996b). “The extended group situation theory (EGST): explaining the amount of change," in Understanding Group Behavior, Vol. 1, eds E. H. Witte and J. H. Davis (Mahwah: Erlbaum), 253-291.

Witte, E. H. (2005). "Theorienentwicklung und -konstruktion in der Sozialpsychologie [Theory development and theory construction in social psychology]," in Entwicklungsperspektiven der Sozialpsychologie [Developmental Perspectives of Social Psychology], ed. E. H. Witte (Lengerich: Pabst), $172-188$.

Witte, E. H., and Davis, J. H. (eds) (1996). Understanding Group Behavior, Vol. 1 and 2. Mahwah: Erlbaum.

Witte, E. H., and Heitkamp, I. (2006). Quantitative rekonstruktionen (retrognosen) als instrument der theorienbildung und theorienprüfung in der sozialpsychologie [Quantitative reconstructions (retrognoses) as an instrument for theory construction and theory assessment in social psychology]. Z. Sozialpsychol. 37, 205-214. doi: 10.1024/0044-3514.37. 3.205

Witte, E. H., and Kaufman, J. (1997). The Stepwise Hybrid Statistical Inference Strategy: FOSTIS. HAFOS, 18. Available at: http://psydok.sulb. uni-saarland.de/frontdoor.php?source_opus $=2286 \&$ la $=$ de [accessed February 22, 2017].

Witte, E. H., and Strohmeier, C. E. (2013). Forschung in der psychologie. Ihre disziplinäre matrix im vergleich zu physik, biologie und sozialwissenschaft [Research in psychology. Its disciplinary matrix as compared to physics, biology, and the social sciences]. Psychol. Rundsch. 64, 16-24. doi: 10.1026/ 0033-3042/a0000145

Witte, E. H., and Zenker, F. (2016a). Beyond schools-reply to Marsman, Ly \& Wagenmakers. Basic Appl. Soc. Psychol. 38, 313-317. doi: 10.1080/01973533. 2016.1227710

Witte, E. H., and Zenker, F. (2016b). Reconstructing recent work on macro-social stress as a research program. Basic Appl. Soc. Psychol. 38, 301-307. doi: 10.1080/ 01973533.2016.1207077

Witte, E. H., and Zenker, F. (2017). Extending a multilab preregistered replication of the ego-depletion effect to a research program. Basic Appl. Soc. Psychol. 39, 74-80. doi: 10.1080/01973533.2016.1269286

Conflict of Interest Statement: The authors declare that the research was conducted in the absence of any commercial or financial relationships that could be construed as a potential conflict of interest.

Copyright (c) 2017 Witte and Zenker. This is an open-access article distributed under the terms of the Creative Commons Attribution License (CC BY). The use, distribution or reproduction in other forums is permitted, provided the original author(s) or licensor are credited and that the original publication in this journal is cited, in accordance with accepted academic practice. No use, distribution or reproduction is permitted which does not comply with these terms. 